\title{
Relação espacial entre o estojo córneo e a falange distal em éguas Campolina adultas com e sem obesidade
}

[Spatial relationship between the hoof capsule and the distal phalanx in adult https://orcid.org/0000-0002-6211-4965 Campolina mares with and without obesity]

\author{
J.F. Magalhães ${ }^{1}$, L.R. Lima ${ }^{1}$, C.F.R. Paz ${ }^{1}$, S.S. Rocha Júnior ${ }^{1}$, A.P.L. Oliveira ${ }^{1}$ \\ P.C. Duarte1, G.E.S. Alves ${ }^{1}$, R.R. Faleiros ${ }^{2 *}$ \\ ${ }^{1}$ Escola de Veterinária - Universidade Federal de Minas Gerais - Belo Horizonte, MG \\ ${ }^{2}$ Pesquisador do CNPq e FAPEMIG
}

\begin{abstract}
RESUMO
A laminite endocrinopática tem acometido um número crescente de equinos com sinais de obesidade. Em um estudo recente com fêmeas jovens (até cinco anos) da raça Campolina, demonstraram-se indícios de alterações no dígito, ainda discretas, aparentemente associadas ao aumento da adiposidade. Com a hipótese de que essa associação é mais evidente em animais em faixa etária superior, o objetivo do presente estudo foi estudar éguas adultas (acima de cinco anos) da raça Campolina com e sem obesidade, avaliando-se radiograficamente a relação espacial entre estojo córneo e falange distal. Foram utilizadas 27 éguas entre seis e 14 anos de idade, sendo analisadas variáveis de adiposidade e medidas radiográficas dos cascos dos membros torácicos de equinos com escore corporal de 5 a 7/9 (grupo controle) e de 8 a 9 (grupo obeso). Foram feitas comparações entre os grupos e correlacionaram-se as variáveis de adiposidade com variáveis casco. A distância de afundamento da falange distal foi cerca de $20 \%$ superior nas éguas obesas $(12,3 \pm 2,5$ contra $10,2 \pm 2,2 \mathrm{~mm}$ no grupo controle). Esse parâmetro também correlacionou $(\mathrm{P}<0,01)$ com vários parâmetros de adiposidade, com destaque para o escore de condição corporal $(r=0,47)$ e a circunferência de pescoço a $75 \%(r=0,42)$. Os resultados corroboram estudos prévios que demonstraram associação entre obesidade e indícios de separação entre falange distal e estojo córneo em equinos de raças nacionais, comprovando, assim, a utilidade da avaliação radiográfica nesses animais. Em conclusão, éguas da raça Campolina com obesidade possuem alterações evidentes na relação espacial entre estojo córneo e falange distal, que se intensificam com o aumento da idade e da adiposidade.
\end{abstract}

Palavras-chave: equino, afundamento, laminite

\begin{abstract}
In a recent study with young females (up to 5 years old) of the Campolina breed, there were discrete indications of alterations in the digit, apparently associated to the increase in adiposity. With the hypothesis that this association is more evident in horses in the upper age group, the objective was to study Campolina adult mares (above 5 years) with and without obesity, evaluating radiographically the spatial relationship between the horn and distal phalanx. Eighteen mares between six and fourteen years and with a body score of 5 to 7/9 (Control Group) and 8 to 9 (Obeso Group) were used. Adiposity and radiographic measurements from the forelimbs were analyzed. Comparisons were made between groups and adiposity variables were correlated with hoof variables. The sinking distance of the distal phalanx was about $20 \%$ higher in obese mares $(12.3 \pm 2.5$ versus $10.2 \pm 2.2 \mathrm{~mm})$. This parameter also correlated $(P<0.01)$ with several adiposity parameters, with emphasis on body score condition $(r=0.47)$ and neck circumference at $75 \%(r=0.42)$. In conclusion, Campolina mares with obesity have obvious alterations in the spatial relation between the corneal and the distal phalanx, which get intense according to increases in age and adiposity.
\end{abstract}

Keywords: equine, sinking, laminitis

Recebido em 6 de abril de 2018

Aceito em 24 de janeiro de 2019

* Autor para correspondência (corresponding author)

E-mail: faleirosufmg@gmail.com 


\section{INTRODUÇÃO}

$\mathrm{Na}$ atualidade, a síndrome metabólica, uma série de alterações endócrinas associadas ao aumento da adiposidade e à disfunção insulínica, é considerada a principal causa de laminite em equinos. Ao contrário da laminite decorrente de quadro séptico, essa forma endocrinopática cursa de modo subclínico, havendo alterações importantes nas estruturas do casco, antes do aparecimento dos sinais clínicos (Frank et al., 2010). Assim, o reconhecimento precoce de tais alterações tem sido considerado como fundamental para o diagnóstico da síndrome metabólica e para o estabelecimento de medidas terapêuticas e nutricionais que impeçam que a laminite endocrinopática evolua para sua fase clínica (Walsh et al., 2009; Frank et al, 2010).

Estudos do grupo Equinova, que buscam associações entre sinais de adiposidade e alterações digitais características de laminite, têm sido realizados em raças nacionais, como o Crioulo, o Mangalarga Marchador e mestiços de Brasileiro de Hipismo. Foram encontradas diversas correlações positivas e significativas entre aumento da adiposidade e alterações das mensurações radiográficas realizadas no dígito desses equinos (Paz et al., 2013; Xavier et al., 2014; Magalhães et al., 2014). Recentemente, realizou-se um estudo com fêmeas jovens (até cinco anos de idade) da raça Campolina. Nesse estudo observaram-se correlações significativas entre obesidade e distância entre a falange distal e o estojo córneo, contudo a associação não foi caracterizada, uma vez que não houve diferenças estatísticas entre os grupos normal e obeso (Magalhães et al., 2017). Com base nesses resultados, os autores sugeriram que tais diferenças seriam mais evidentes em equinos Campolina em faixas etárias superiores, que estariam expostos por mais tempo aos malefícios do excesso de adiposidade.

Com a hipótese de que as alterações de casco devido à obesidade são evidentes em éguas Campolina adultas, o objetivo da presente pesquisa foi estudar, por meio de avaliação radiográfica, a relação espacial entre o estojo córneo e a falange distal de éguas adultas (acima de cinco anos de idade).

\section{MATERIAL E MÉTODOS}

A metodologia utilizada foi aprovada pelo Comitê de Ética (Ceua), certificado 278 / 2014. Foram avaliados cascos dos membros torácicos de fêmeas adultas da raça Campolina, oriundas de diferentes criatórios. Inicialmente as éguas passaram por exame clínico em estática e dinâmica e pelo exame da pinça de casco (Stashak et al, 2002), sendo excluídas aquelas que apresentavam claudicação e sensibilidade dolorosa no casco.

Selecionaram-se, para o estudo, 27 éguas adultas (de seis a 14 anos), que foram avaliadas quanto ao escore de condição corporal (ECC) de acordo com Henneke et al. (1983), que varia de 1 a 9 (1= animal emaciado e 9= animal extremamente obeso). Essa avaliação, assim como as outras avaliações por escore, era sempre realizada por dois avaliadores para diminuir a possibilidade de erros. Com base nessa classificação, foram divididos em dois grupos, o grupo controle (ECC de 5 a 7) e o grupo obeso (ECC 8 a 9). O grupo controle foi composto de 13 éguas (sete oriundas de haras), e o grupo obeso de 14 éguas (todas oriundas da Exposição Nacional da Raça).

A fim de se compararem os grupos quanto às características físicas e de adiposidade, registraram-se o peso e a altura de cada um dos animais por meio de fita de pesagem e hipômetro (WalMur Instrumentos Veterinários). Para verificação da deposição de gordura na face dorsal (crista) do pescoço, que constitui um sinal de doença metabólica, foi utilizado um sistema de escore para adiposidade no pescoço (EAP) descrito por Carter et al. (2009), que varia de 0 a $5(0=$ crista não visível nem palpável e $5=$ crista tão grande que se inclina permanentemente para um dos lados).

Medidas da circunferência do pescoço foram tomadas em três alturas diferentes em relação ao comprimento total do pescoço, com auxílio de fita métrica, segundo metodologia prévia (Frank et al., 2006). Essas medidas iniciaram-se em pontos dorsais que correspondiam a $25 \%, 50 \%$ e $75 \%$ desse comprimento. Para a avaliação radiográfica da relação espacial entre falange distal e estojo córneo, ambos os cascos dos membros torácicos foram limpos e posicionados sobre dois tocos de madeira, de igual altura, e radiografados na projeção lateromedial, a uma 
distância padronizada, usando-se um aparelho de raios X (EcoRay CO., Ltd 1060HF - Korea) dotado de distanciador com iluminação a laser.

As exposições foram realizadas sempre com $80 \mathrm{Kv}$ e $2,5 \mathrm{mAs}$. Para possibilitar a identificação radiográfica da superfície dorsal da parede do casco e do plano da banda coronária, um fio metálico de comprimento conhecido foi aderido à superfície dorsal da pinça do casco, com sua extremidade proximal sempre coincidindo com o plano da banda coronária. Esse fio metálico foi posicionado sempre pela mesma pessoa. Cada casco foi radiografado duas vezes, portanto quatro imagens foram obtidas de cada animal.

As imagens foram digitalizadas por meio de um sistema computadorizado, e as mensurações feitas por meio do programa computacional Metron Hoof-Pro ${ }^{4}$. O comprimento do marcador radiopaco foi utilizado para corrigir o fator de ampliação. As quatro imagens de cada animal foram aferidas, e o resultado final de cada membro foi a média das duas imagens de cada um dos membros.

De acordo com o programa Metron Hoof-Pro ${ }^{4}$ e conforme descrito por Thrall (2002) e Pollitt (2008), foram obtidas as medidas para as seguintes variáveis: distância entre os planos horizontais entre a banda coronária e o ápice do processo extensor da falange distal (afundamento), ângulo palmar, profundidade ou espessura de sola, ângulo da parede dorsal do casco (Ang. Casco), distâncias entre as superfícies dorsais da falange distal e da parede do casco, proximal (CF Prox.) e distal (CF Distal), ângulo do eixo casco-quartela (cascoquartela), ângulo entre as superfícies dorsais da falange distal e da parede do casco (rotação) e breakover. Ilustrações sobre tais medidas estão disponíveis em trabalho prévio (Magalhães et al., 2017).

Incialmente, compararam-se, dentro do grupo controle, os dados obtidos entre equinos oriundos da exposição e do haras, utilizando-se o teste $\mathrm{t}$ de Student para variáveis paramétricas e o teste Mann-Whitney para as não paramétricas. Posteriormente, realizou-se a comparação entre grupos, utilizando-se o teste $\mathrm{t}$ de Student para variáveis paramétricas e o teste Mann-Whitney para as não paramétricas. Para as medidas de correlação, empregou-se o teste de Pearson para duas amostras paramétricas e o teste de Spearman para comparações que envolvam pelo menos uma variável não paramétrica. Em todos os testes, considerou-se $\mathrm{P}<0,05$. Para efeito de comparação dos graus de correlação, consideraram-se os seguintes níveis de correlação: elevada $(>0,60)$, média (de 0,30 a $0,60)$ e baixa $(<0,30)$.

\section{RESULTADOS E DISCUSSÃO}

Os valores referentes às variáveis de adiposidade estão representados na Tab. 1. Apesar de não haver diferença significativa entre o peso de éguas do grupo controle e de éguas do grupo obeso, o grupo obeso apresentou maior ECC $(\mathrm{P}<0,0001)$ e maior EAP $(\mathrm{P}=0,004)$, demonstrando maior deposição de gordura, de forma bem evidente na região dorsal do pescoço, em éguas adultas da raça Campolina.

Todas as éguas obesas apresentaram EAP acima de 3, o que está associado ao maior risco de desenvolvimento de doenças metabólicas e laminite (Walsh et al., 2009; Frank et al, 2010). Esse resultado também foi encontrado por Magalhães et al. (2017) ao estudarem fêmeas Campolina jovens (até cinco anos). Corroborando ainda com esses achados, foram encontradas correlações significativas entre teor de insulina e EAP e ECC em éguas da raça Crioula (Paz et al., 2013).

Os valores das variáveis radiográficas dos cascos estão na Tab. 2. A variável que mais se destacou na comparação entre os grupos foi a distância de afundamento $(\mathrm{P}=0,003)$. As éguas obesas tiveram uma distância de afundamento média $(12,3 \pm 2,5 \mathrm{~mm})$ cerca de $20 \%$ superior à média do grupo controle $(10,2 \pm 2,2 \mathrm{~mm})$. Uma vez que essa variável não sofre interferência do casqueamento (Eustace, 2010), é considerada como uma das mais efetivas para avaliar a movimentação da falange distal.

Paz et al. (2013) publicaram o primeiro estudo controlado a demonstrar que equinos obesos, mesmo sem apresentar nenhum histórico ou sinal clínico de laminite, estão mais predispostos a apresentar alterações no posicionamento da falange distal. Magalhães et al. (2014), quando avaliaram éguas da raça Mangalarga Marchador, também encontraram uma distância de afundamento maior $(11,7 \%)$ nas éguas obesas ao 
serem comparadas com as éguas do grupo normal. Ainda neste mesmo ano, Xavier et al. (2014) encontraram resultados que sugerem a existência de correlação entre obesidade e sinais de laminite em equinos de patrulhamento. Esses trabalhos corroboram os resultados encontrados no presente trabalho com éguas Campolina.

Tabela 1. Valores mínimo, máximo, média, desvio-padrão e valor de P na comparação de variáveis físicas e de adiposidade de éguas Campolina adultas com (grupo controle, $n=27$ cascos) e sem (grupo obeso, $n=$ 26 cascos) obesidade

\begin{tabular}{llllllllll}
\hline \multicolumn{3}{c}{ Grupo controle } & \multicolumn{7}{c}{ Grupo obeso } \\
Variável & Mín & Máx & Média & $\begin{array}{l}\text { Desvio- } \\
\text { padrão }\end{array}$ & Mín & Máx & Média & $\begin{array}{l}\text { Desvio- } \\
\text { padrão }\end{array}$ & $\begin{array}{l}\text { Valor } \\
\text { de P* }\end{array}$ \\
Idade (anos) & 6 & 13 & 9,57 & 2,9 & 6 & 14 & 7,62 & 2,47 & 0,072 \\
Peso (kg) & 436 & 600 & 524 & 53,59 & 480 & 640 & 548 & 38,5 & 0,201 \\
Altura (m) & 1,49 & 1,62 & 1,56 & 0,04 & 1,51 & 1,67 & 1,57 & 0,04 & 0,289 \\
ECC (grau) & 5 & 7 & 6,5 & 0,76 & 8 & 9 & 8,15 & 0,38 & 0,001 \\
EAP (grau) & 2 & 4 & 2,79 & 0,8 & 3 & 4 & 3,62 & 0,51 & 0,004 \\
CP (cm) & 80 & 103 & 93,8 & 7,4 & 83 & 107 & 91,6 & 7,29 & 0,436 \\
DP 25\% (cm) & 74 & 86,5 & 80,5 & 3,47 & 72 & 83 & 79,1 & 3,48 & 0,312 \\
DP 50\% (cm) & 91 & 104,5 & 97,5 & 3,99 & 92 & 108 & 99,9 & 4,42 & 0,147 \\
DP 75\% (cm) & 106 & 210 & 192 & 8,86 & 111 & 133 & 121 & 4,96 & 0,105 \\
\hline
\end{tabular}

Mín = mínimo. Máx = máximo. $\mathrm{ECC}=$ escore de condição corporal. $\mathrm{EAP}=$ escore de adiposidade de pescoço. $\mathrm{CP}=$ comprimento de pescoço. DP = diâmetro de pescoço. * Teste t de Sudent para variáveis paramétricas e teste de Mann-Whitney para as não paramétricas.

Todos os animais dos trabalhos citados acima, como as éguas do presente estudo, não apresentavam sinais clínicos de laminite, apesar de os animais do grupo obeso terem alguma alteração radiográfica no posicionamento da falange distal. Os valores preconizados como normais pela literatura internacional para a distância de afundamento da falange distal são inferiores a $10 \mathrm{~mm}$ (Eustace, 2010) ou a $11 \mathrm{~mm}$ (Pollitt, 2008). Uma vez que a distância média de afundamento nas éguas do grupo obeso foi superior às referências e com base em estudos prévios que demonstraram alteração na relação espacial entre falange distal e estojo córneo em animais com obesidade e distúrbio metabólico (Kariskoski et al., 2015), considerou-se que esse fato se relaciona a alterações endócrinas causadas pelo excesso de adiposidade, as quais promovem alterações nas lâminas do casco, com enfraquecimento delas, o que permitiria movimentação da falange no sentido distal. Esse fato também foi comprovado quando da indução de hiperglicemia em equinos Puro Sangue Inglês
(PSI), o que resultou em alterações histológicas importantes, sem que os animais apresentassem claudicação (Walsh et al., 2009; De Laat et al., 2012).

Em contraste com o presente estudo, éguas jovens da raça Campolina não apresentaram diferenças significativas para distância de afundamento (Magalhães et al., 2017). Tais discrepâncias podem ser explicadas pelos efeitos prolongados da obesidade em fêmeas adultas. Estudos em humanos têm demonstrado que pessoas que se tornam obesas durante a infância e adolescência têm maior chance de apresentar vários problemas médicos durante a fase adulta (Maffeis e Tatò, 2001). Assim, os achados do presente estudo indicam que éguas Campolina, que recebem dieta acima de suas necessidades fisiológicas, tornando-se obesas, apresentam alterações radiográficas de casco indicadoras de laminite, que se manifestam a partir dos cinco anos de idade. 
Relação espacial...

Tabela 2. Valores mínimo, máximo, média, desvio-padrão e valor de $\mathrm{P}$ na comparação pelo teste $\mathrm{t}$ de Student de variáveis radiográficas dos cascos de éguas Campolina adultas com (grupo controle, $n=27$ cascos) e sem (grupo obeso, $n=26$ cascos) obesidade

\begin{tabular}{|c|c|c|c|c|c|c|c|c|c|}
\hline & Grupo & contro & & & Grupc & obeso & & & \\
\hline Variável & Mín & Máx & Média & $\begin{array}{l}\text { Desvio- } \\
\text { padrão }\end{array}$ & Mín & Máx & Média & $\begin{array}{l}\text { Desvio- } \\
\text { padrão }\end{array}$ & $\begin{array}{l}\text { Valor } \\
\text { de P }\end{array}$ \\
\hline Ângulo Palmar $\left(^{\circ}\right)$ & 0,22 & 10,7 & 4,51 & 2,78 & $-2,81$ & 6,6 & 2,11 & 2,38 & 0,001 \\
\hline Afundamento (mm) & 6,5 & 14,3 & 10,2 & 2,2 & 6,4 & 17,1 & 12,3 & 2,5 & 0,003 \\
\hline $\begin{array}{l}\text { Espessura de Sola } \\
(\mathrm{mm})\end{array}$ & 11,3 & 27,7 & 17,4 & 3,9 & 14,9 & 27,4 & 20,9 & 3,9 & 0,002 \\
\hline $\begin{array}{l}\text { Âng. Dorsal do } \\
\text { Casco }\left(^{\circ}\right)\end{array}$ & 43,4 & 59,7 & 51,3 & 4,1 & 42,1 & 55,6 & 49,6 & 3,09 & 0,089 \\
\hline $\begin{array}{l}\text { Dist. CF Proximal } \\
(\mathrm{mm})\end{array}$ & 15,4 & 21,7 & 18,5 & 1,7 & 15,4 & 22,4 & 18,9 & 1,7 & 0,393 \\
\hline $\begin{array}{l}\text { Dist. CF Distal } \\
(\mathrm{mm})\end{array}$ & 13,5 & 19,9 & 17 & 1,7 & 14,1 & 20,6 & 17 & 1,6 & 0,926 \\
\hline $\begin{array}{l}\text { Suporte de pinça } \\
(\%)\end{array}$ & 61,6 & 83,8 & 70,4 & 6,06 & 64,6 & 84,7 & 73,5 & 5,66 & 0,063 \\
\hline $\begin{array}{l}\text { Âng. Inter. Distal } \\
\left({ }^{\circ}\right)\end{array}$ & 0,49 & 31,2 & 12,5 & 8,34 & 5,69 & 32,4 & 23,0 & 6,83 & 0,001 \\
\hline $\begin{array}{l}\text { Âng. Inter. } \\
\text { Proximal }\left(^{\circ}\right)\end{array}$ & $-5,96$ & 9,26 & 2,19 & 4,59 & $-8,68$ & 7,26 & 2,01 & 3,69 & 0,878 \\
\hline $\begin{array}{l}\text { Comprimento P2 } \\
\text { (cm) }\end{array}$ & 3,64 & 4,98 & 4,3 & 0,37 & 3,52 & 4,17 & 3,92 & 0,18 & 0,001 \\
\hline Âng. Rotação P3 $\left(^{\circ}\right)$ & $-6,19$ & 0,73 & $-2,27$ & 1,81 & $-5,28$ & $-0,42$ & $-2,78$ & 1,15 & 0,230 \\
\hline $\begin{array}{l}\text { Âng. } \\
\text { Casco/quartela }\left(^{\circ}\right)\end{array}$ & 155 & 183 & 169 & 7,16 & 152 & 173 & 161 & 5,68 & 0,001 \\
\hline $\begin{array}{l}\text { Dist. de breakover } \\
(\mathrm{cm})\end{array}$ & 2,55 & 4,09 & 3,08 & 0,44 & 2,43 & 4,33 & 3,29 & 0,51 & 0,123 \\
\hline
\end{tabular}

Mín = mínima. Máx = máxima. Dist. = distância. $\mathrm{CF}=$ casco/falange. Âng. = ângulo. Inter. = interfalângica.

Ainda na Tab. 2, outros resultados estatisticamente diferentes foram ângulo palmar $(\mathrm{P}=0,001)$ e espessura de sola $(\mathrm{P}=0,002)$. Os animais obesos apresentaram ângulo palmar menor $(2,11 \pm 2,38$ graus $)$ e espessura de sola maior $(20,9 \pm 3,9 \mathrm{~mm})$ do que os do grupo controle $(4,51 \pm 2,78$ graus e $17,4 \pm 3,9 \mathrm{~mm}$, respectivamente). Tais achados foram atribuídos à ocorrência de casqueamento e ferragemento mais frequente em animais do grupo obeso do que do grupo controle. O ângulo palmar é uma das principais mensurações para avaliar rotação distopalmar da falange distal. De acordo com a literatura internacional, a variação normal é de 3 a $5^{\circ}$ (Redden, 2003; O’Grady, 2008). Esse ângulo pode sofrer grande variação com diferentes práticas de casqueamento. Kummer et al. (2006) encontraram uma porcentagem de alteração do ângulo palmar de -30 a $-40 \%$ após o casqueamento.
No caso do ângulo da parede dorsal do casco, na literatura internacional há relato de valor de normalidade de $52,2^{\circ} \pm 3,7^{\circ}$ (Dyson et al., 2011). As éguas Campolina avaliadas apresentaram médias inferiores tanto no grupo obeso $\left(49,61^{\circ} \pm 3,09^{\circ}\right) \quad$ quando no controle $\left(50,74^{\circ} \pm 4,2^{\circ}\right)$. Esse ângulo também pode sofrer grande interferência por casqueamento (Sherlock e Parks, 2013). Kummer et al. (2006) encontraram uma porcentagem de alteração do ângulo do casco de -5 a $6 \%$ após casqueamento.

A distância proximal encontrada entre o casco e a falange das éguas Campolina foi em torno de $18 \mathrm{~mm}$, e a distal em torno de $17 \mathrm{~mm}$. Esse achado foi semelhante ao encontrado para fêmeas Campolina jovens (Magalhães et al., 2017). Além disso, esses valores estão próximos aos encontrados por Magalhães et al. (2014) e Xavier et al. (2014), que foram em média $19 \mathrm{~mm}$ e $17 \mathrm{~mm}$, respectivamente. 
Na Tab. 3, estão os índices de correlações entre as variáveis de adiposidade e as variáveis de morfometria do casco. Novamente, a variável que mais se destacou foi a distância de afundamento, que se correlacionou estatisticamente com vários parâmetros de adiposidade, como o ECC $(\mathrm{r}=0,47)$, o EAP $(0,34)$ e as circunferências de pescoço tomadas a $25 \%(r=0,28)$, a $50 \%(r=0,30)$ e $75 \%(r=0,42)$ de seu comprimento. Além disso, também existiu correlação positiva entre a distância cascofalange $(\mathrm{CF})$ e variáveis de adiposidade. Tais achados reforçam as considerações de que as alterações endócrinas que sabidamente promovem enfraquecimento do tecido lamelar do casco e afastamento entre a parede dorsal do casco e a falange distal se manifestam mais intensamente em éguas Campolina adultas, que têm maior exposição aos malefícios da obesidade do que éguas jovens.

Tabela 3. Índices de correlação (r) e respectivos valores de $\mathrm{P}$ entre variáveis físicas e de adiposidade e variáveis morfométricas de casco em éguas adultas da raça Campolina

\begin{tabular}{|c|c|c|c|c|c|c|c|c|c|c|}
\hline & & $\begin{array}{l}\text { Ang. } \\
\text { Palm. }\end{array}$ & $\begin{array}{l}\text { Dist. } \\
\text { Afun. }\end{array}$ & $\begin{array}{l}\text { Esp. } \\
\text { Sola }\end{array}$ & $\begin{array}{l}\text { Ang. } \\
\text { Casco }\end{array}$ & $\begin{array}{l}\text { CF } \\
\text { Prox } \\
\end{array}$ & $\begin{array}{l}\text { CF } \\
\text { Dist. }\end{array}$ & $\begin{array}{l}\text { Ang. } \\
\text { Rot. }\end{array}$ & $\begin{array}{l}\text { Ang. } \\
\text { C/Q }\end{array}$ & $\begin{array}{l}\text { Breako } \\
\text { ver }\end{array}$ \\
\hline Idade & $\begin{array}{l}\mathrm{r} \\
\mathrm{P}\end{array}$ & $\begin{array}{l}0,14 \\
0,32\end{array}$ & $\begin{array}{l}-0,21 \\
0,13\end{array}$ & $\begin{array}{l}-0,10 \\
0,48\end{array}$ & $\begin{array}{l}-0,16 \\
0,24\end{array}$ & $\begin{array}{l}-0,16 \\
0,24\end{array}$ & $\begin{array}{l}-0,10 \\
0,47\end{array}$ & $\begin{array}{l}-0,04 \\
0,80\end{array}$ & $\begin{array}{l}0,24 \\
0,10\end{array}$ & $\begin{array}{l}0,10 \\
0,50\end{array}$ \\
\hline ECC & $\begin{array}{l}\mathrm{r} \\
\mathrm{P}\end{array}$ & $\begin{array}{l}-0,36 \\
0,01 \\
* *\end{array}$ & $\begin{array}{l}0,47 \\
\mathrm{P}<0,01 \\
* * *\end{array}$ & $\begin{array}{l}0,46 \\
\mathrm{P}<0,01 \\
* * *\end{array}$ & $\begin{array}{l}-0,11 \\
0,41\end{array}$ & $\begin{array}{l}0,19 \\
0,16\end{array}$ & $\begin{array}{l}-0,02 \\
0,90\end{array}$ & $\begin{array}{l}-0,31 \\
0,02 \\
*\end{array}$ & $\begin{array}{l}-0,60 \\
\mathrm{P}<0,01 \\
* * *\end{array}$ & $\begin{array}{l}0,22 \\
0,10\end{array}$ \\
\hline Peso & $\begin{array}{l}r \\
P\end{array}$ & $\begin{array}{l}-0,11 \\
0,42\end{array}$ & $\begin{array}{l}0,44 \\
\mathrm{P}<0,01 \\
* * *\end{array}$ & $\begin{array}{l}0,22 \\
0,10\end{array}$ & $\begin{array}{l}0,19 \\
0,17\end{array}$ & $\begin{array}{l}0,52 \\
\mathrm{P}<0,0 \\
1 \\
* * *\end{array}$ & $\begin{array}{l}0,21 \\
0,13\end{array}$ & $\begin{array}{l}-0,29 \\
0,03 \\
*\end{array}$ & $\begin{array}{l}-0,21 \\
0,12\end{array}$ & $\begin{array}{l}0,10 \\
0,60\end{array}$ \\
\hline Altur & $\begin{array}{l}\mathrm{r} \\
\mathrm{P}\end{array}$ & $\begin{array}{l}-0,20 \\
0,14\end{array}$ & $\begin{array}{l}0,33 \\
0,01 \\
*\end{array}$ & $\begin{array}{l}0,13 \\
0,32\end{array}$ & $\begin{array}{l}-0,04 \\
0,76\end{array}$ & $\begin{array}{l}0,28 \\
0,03 \\
*\end{array}$ & $\begin{array}{l}0,16 \\
0,24\end{array}$ & $\begin{array}{l}0,06 \\
0,64\end{array}$ & $\begin{array}{l}-0,29 \\
0,03 \\
*\end{array}$ & $\begin{array}{l}0,14 \\
0,29\end{array}$ \\
\hline EAP & $\begin{array}{l}r \\
P\end{array}$ & $\begin{array}{l}-0,34 \\
0,01 \\
*\end{array}$ & $\begin{array}{l}0,34 \\
0,01 \\
* *\end{array}$ & $\begin{array}{l}0,66 \\
\mathrm{P}<0,01 \\
* * *\end{array}$ & $\begin{array}{l}-0,05 \\
0,74\end{array}$ & $\begin{array}{l}0,19 \\
0,17\end{array}$ & $\begin{array}{l}-0,10 \\
0,67\end{array}$ & $\begin{array}{l}-0,25 \\
0,06\end{array}$ & $\begin{array}{l}-0,55 \\
\mathrm{P}<0,01 \\
* * *\end{array}$ & $\begin{array}{l}0,46 \\
\mathrm{P}<0,01 \\
* * *\end{array}$ \\
\hline $\begin{array}{l}\text { CompP } \\
\text { esc. }\end{array}$ & $\begin{array}{l}r \\
P\end{array}$ & $\begin{array}{l}0,13 \\
0,35\end{array}$ & $\begin{array}{l}0,26 \\
0,05 \\
*\end{array}$ & $\begin{array}{l}-0,31 \\
0,02 \\
*\end{array}$ & $\begin{array}{l}-0,02 \\
0,90\end{array}$ & $\begin{array}{l}0,34 \\
0,01 \\
* *\end{array}$ & $\begin{array}{l}0,35 \\
0,01 \\
* *\end{array}$ & $\begin{array}{l}-0,01 \\
0,93\end{array}$ & $\begin{array}{l}0,11 \\
0,43\end{array}$ & $\begin{array}{l}0,12 \\
0,36\end{array}$ \\
\hline $\begin{array}{l}\text { Circ. } \\
25 \%\end{array}$ & $\begin{array}{l}\mathrm{r} \\
\mathrm{P}\end{array}$ & $\begin{array}{l}0,13 \\
0,33\end{array}$ & $\begin{array}{l}0,28 \\
0,04 \\
*\end{array}$ & $\begin{array}{l}0,02 \\
0,91\end{array}$ & $\begin{array}{l}0,32 \\
0,01 \\
*\end{array}$ & $\begin{array}{l}0,37 \\
0,01 \\
* *\end{array}$ & $\begin{array}{l}0,19 \\
0,15\end{array}$ & $\begin{array}{l}-0,20 \\
0,15\end{array}$ & $\begin{array}{l}-0,10 \\
0,54\end{array}$ & $\begin{array}{l}0,03 \\
0,83\end{array}$ \\
\hline $\begin{array}{l}\text { Circ. } \\
50 \%\end{array}$ & $\begin{array}{l}r \\
P\end{array}$ & $\begin{array}{l}-0,12 \\
0,36\end{array}$ & $\begin{array}{l}0,30 \\
0,02 \\
*\end{array}$ & $\begin{array}{l}0,32 \\
0,02 \\
*\end{array}$ & $\begin{array}{l}0,21 \\
0,12\end{array}$ & $\begin{array}{l}0,35 \\
0,01 \\
* *\end{array}$ & $\begin{array}{l}0,07 \\
0,63\end{array}$ & $\begin{array}{l}-0,29 \\
0,03 \\
*\end{array}$ & $\begin{array}{l}-0,29 \\
0,03 \\
*\end{array}$ & $\begin{array}{l}0,11 \\
0,42\end{array}$ \\
\hline $\begin{array}{l}\text { Circ. } \\
75 \%\end{array}$ & $\begin{array}{l}\mathrm{r} \\
\mathrm{P}\end{array}$ & $\begin{array}{l}-0,04 \\
0,75\end{array}$ & $\begin{array}{l}0,42 \\
0,01 \\
* *\end{array}$ & $\begin{array}{l}0,30 \\
0,03 \\
*\end{array}$ & $\begin{array}{l}0,27 \\
0,04 \\
*\end{array}$ & $\begin{array}{l}0,52 \\
\mathrm{P}<0,0 \\
1 \\
* * *\end{array}$ & $\begin{array}{l}0,12 \\
0,38\end{array}$ & $\begin{array}{l}-0,40 \\
0,01 \\
* *\end{array}$ & $\begin{array}{l}-0,34 \\
0,01 \\
* *\end{array}$ & $\begin{array}{l}0,10 \\
0,54\end{array}$ \\
\hline
\end{tabular}

$\mathrm{r}>0,60=$ correlação alta; $\mathrm{r} 0,30$ a $0,60=$ correlação média; $r<0,30=$ correlação baixa.

Uma limitação do presente estudo foi a ausência de análise da disfunção insulínica nos equinos utilizados. Restrições de tempo e dificuldades na obtenção de anuência por parte dos proprietários foram fatores preponderantes, visto que a maioria dos animais foram avaliados durante a exposição nacional da raça. Entretanto, muitos trabalhos já demonstraram a relação entre obesidade e resistência insulínica em cavalos. Sabe-se, também, que a captação de glicose pelas lâminas do casco não é dependente de insulina
(Asplin et al., 2007; Walsh et al., 2009), portanto a fragilidade de conexão entre falange distal e estojo córneo não deve ser causada pela falta de glicose. Hipóxia celular promovida por ações vasorreguladoras da insulina (Asplin et al., 2007; Frank et al., 2010) e alterações fenotípicas nos queratinócitos decorrentes da ativação de receptores do fator de crescimento semelhante à insulina (IGF1), por elevadas concentrações de insulina circulante, têm sido mencionadas como fatores desencadeadores da laminite 
endocrinopática associada à obesidade em equinos.

Contrariamente ao aqui relatado, não houve correlação entre a distância de afundamento com as variáveis de adiposidade em éguas jovens da raça Campolina (Magalhães et al. (2017). Tais achados também reforçam a noção de que os efeitos deletérios da doença metabólica sobre o casco se manifestam após um tempo maior de exposição dos equinos ao excesso de adiposidade.

Corroborando com os presentes resultados, estudos prévios do grupo de pesquisa Equinova têm verificado, de forma consistente, correlações positivas e associações entre parâmetros de adiposidade e alterações radiográficas que indicam $o$ enfraquecimento da união entre $o$ estojo córneo em equinos obesos de raças nacionais (Paz et al., 2013; Magalhães et al., 2014; Xavier et al., 2014; Magalhães et al., 2017). Uma vez que todos esses estudos foram realizados em equinos sem histórico ou sintomas presentes de laminite, tais achados, quando analisados em conjunto, demonstram que $o$ exame radiográfico periódico da falange distal pode ser um procedimento valioso no diagnóstico precoce, na monitoração da evolução da doença e na determinação de risco do desenvolvimento de laminite clínica em equinos de raças nacionais com sobrepeso e obesidade.

\section{CONCLUSÕES}

Éguas adultas da raça Campolina com obesidade apresentam alterações na relação espacial entre o estojo córneo e a falange distal, com destaque para aumento da distância de afundamento. A intensidade dessas alterações ocorre de forma proporcional ao aumento do acúmulo de gordura subcutânea, principalmente na região do pescoço. Tais achados corroboram achados prévios em Campolinas jovens e em outras raças nacionais e indicam a presença de doença metabólica associada à laminite subclínica, com alterações mais evidentes se manifestando com o avançar da idade.

\section{AGRADECIMENTOS}

CNPq, FAPEMIG, CAPES e Colegiado de PósGraduação em Ciência Animal da UFMG pelo apoio financeiro.

\section{REFERÊNCIAS}

ASPLIN, K.E.; SILLENCE, M.N.; POLLITT, C.C.; MCGOWAN, C.M. Induction of laminitis by prolonged hyperinsulinaemia in clinically normal ponies. Vet. J., v.174, p.530-535, 2007.

CARTER, R.A.; GEOR, R.J.; BURTON STANIAR, W. et al. Apparent adiposity assessed by standardised scoring systems and morphometric measurements in horses and ponies. Vet. J., v.179, p.204-210, 2009.

DE LAAT, M.A.; SILLENCE, M.N.; McGOWAN, C.M.; POLLITT C.C. Continuous intravenous infusion of glucose induces endogenous hyperinsulinaemia and lamellar histopathology in Standardbred horses. Vet. J., v.191, p.317-322, 2012.

DYSON, S.J.; TRANQUILLE, C.A.; COLLINS, S.N. et al. External characteristics of the lateral aspect of the hoof differ between non-lame and lame horses. Vet. J., v.190, p.364-371, 2011.

EUSTACE, R.A. Clinical presentation, diagnosis, and prognosis of chronic laminitis in Europe. Vet. Clin. N. Am. Equine Pract., v.26, p.391-405, 2010.

FRANK, N.; ELLIOTT, S.B.; BRANDT, L.E. et al. Physical characteristics, blood hormone concentrations, and plasma lipid concentrations in obese horses with insulin resistance. $J$. Am. Vet. Med. Assoc., v.228, p.1383-1390, 2006.

FRANK, N.; GEOR, R.J.; BAILEY, S.R. et al. Equine metabolic syndrome. J. Vet. Intern. Med., v. 24, p.467-475, 2010.

HENNEKE, D.R.; POTTER, G.D.; KREIDER, J.L. et al. Relationship between condition score, physical measurements and body fat percentage in mares. Equine Vet. J., v.15, p.371-372, 1983.

KARISKOSKI, N.P.; MCGOWAN, C.M.; SINGER, E.R. et al. Pathology of natural cases of equine endocrinopathic laminitis associated with hyperinsulinemia. Vet. Pathol., v.52, p.945956, 2015.

KUMMER, M.; GEYER, H.; IMBODEN, I. et $a l$. The effect of foot trimming on radiographic measurements of the front feet of normal Warmblood horses. Vet. J., v.172, p.58-66, 2006. 
MAFFEIS, C.; TATÒ, L. Long-term effects of childhood obesity on morbidity and mortality. Horm. Res., v.55, Suppl.1, p.42-45, 2001.

MAGALHÃES, J.F.; LIMA, I.R.; LIMA, L.R. et al. Estudo da correlação de medidas radiográficas indicadoras de laminite em éguas da raça Mangalarga Marchador com e sem sinais de sobrepeso. Arq. Bras. Med. Vet. Zootec., v.66, p.1023-1032, 2014.

MAGALHÃES, J.F.; LIMA, L.R.; PAZ, C.F.R. et al. Relação espacial entre a falange distal e o estojo córneo em éguas Campolinas jovens com e sem sinais de obesidade. Pesqui. Vet. Bras., v.37, p.1025-1031, 2017.

O'GRADY, S.E. Basic farriery for the performance horse. Vet. Clin. N. Am. Equine Pract., v. 24, p.203-218, 2008.

PAZ, C.F.R.; PAGANELA, J.; AMARAL, L.A. et al. Relação entre obesidade, insulina plasmática e posicionamento da falange distal em equinos da raça crioula. Arq. Bras. Med. Vet. Zootec., v.65, p.1699-1705, 2013.

POLLITT, C.C. Equine laminitis current concepts. Queensland- Australia, [s.n.], 2008. 116p. (Publication No. 08/062, project no. RIRDC UQ-118A).
REDDEN, R.F. Clinical and radiographic examination of the equine foot. Proc. Am. Assoc. Equine Practit., v.49, p.169-185, 2003.

SHERLOCK, C.; PARKS, A. Radiographic and radiological assessment of laminitis. Equine Vet. Educ., v.25, p.524-535, 2013.

STASHAK, T.S.; HILL, C.; KLIMESH, R.; OVNICK, G. Trimming and shoeing for balance and soundness. In: STASHAK, T.S. Adams lameness in horses. 5.ed. Philadelphia: Lippincott Williams \& Wilkins, 2002. p.10811142.

THRALL, D.E. Textbook of veterinary diagnostic radiology. 4.ed. Philadelphia: Saunders, 2002. 758p.

WALSH, D.M.; McGOWAN, C.M.; McGOWAN, T. et al. Correlation of plasma insulin concentration with laminitis score in a field study of Equine Cushing's Disease and Equine Metabolic Syndrome. J. Equine Vet. Sci., v.29, p.87-94, 2009.

XAVIER, V.F.; LIMA, I.R.; MARVAL, C.A.D. et al. Estudo radiográfico do posicionamento da falange distal e suas possíveis correlações com obesidade em equinos de patrulhamento da Polícia Militar do Estado de Minas Gerais. Arq. Bras. Med. Vet. Zootec., v.66, p.672-680, 2014. 